\title{
Patrimonialiser, revitaliser, habiter l'industrie en ville : une question politique et sociale vivante plus qu'une simple question de renouveau urbain
}

To make heritage, to revitalize, to live in industrial territories: an alive political

and social question

Die Industriestädte zu Erbe zumachen, wiederzuleben, bewohnen: eine lebendige politische und soziale Frage

Corinne Luxembourg

\section{OpenEdition}

\section{Journals}

Electronic version

URL: http://journals.openedition.org/rge/5105

DOI: $10.4000 /$ rge.5105

ISSN: 2108-6478

\section{Publisher}

Association des géographes de l'Est

Printed version

Date of publication: 30 December 2013

ISSN: 0035-3213

\section{Electronic reference}

Corinne Luxembourg, «Patrimonialiser, revitaliser, habiter l'industrie en ville : une question politique et sociale vivante plus qu'une simple question de renouveau urbain », Revue Géographique de l'Est [Online], vol. 53 / 3-4 | 2013, Online since 06 July 2014, connection on 08 September 2020. URL http://journals.openedition.org/rge/5105; DOI : https://doi.org/10.4000/rge.5105

This text was automatically generated on 8 September 2020

Tous droits réservés 


\section{Patrimonialiser, revitaliser, habiter l'industrie en ville : une question politique et sociale vivante plus qu'une simple question de renouveau urbain}

To make heritage, to revitalize, to live in industrial territories: an alive political and social question

Die Industriestädte zu Erbe zumachen, wiederzuleben, bewohnen: eine lebendige politische und soziale Frage

Corinne Luxembourg

\section{Introduction}

1 Le processus de désindustrialisation touche nombre de villes petites et moyennes qui avaient accueilli les activités de production après la déconcentration des années 1950-1960 et la politique de l'agrément-redevance. Perdant les emplois de leurs habitants, ces villes perdent consécutivement peu à peu une part de leur population, elles rétrécissent (Fol, Cunningham, 2010). La fragmentation du tissu urbain résultant de la désertion des activités industrielles bouleverse durablement le paysage d'une part mais aussi l'urbanité de ces villes entraînant avec eux les représentations qu'en ont les habitants, leur appropriation, leurs images. Il ne s'agit pas dans cet article de différencier les villes industrielles, des villes usines ou des villes minières, ni d'étudier un processus dans ses étapes d'arrêt d'activité, de deuil de l'activité puis de patrimonialisation, mais bien d'étudier la portée politique et sociale de la patrimonialisation dans des territoires industrialisés sur un temps long. En cela la forte spécialisation des villes moyennes rend les processus souvent similaires, 
indépendamment de l'éventuelle existence urbaine préindustrielle. Ces recompositions profondes du territoire urbain impliquent alors des recompositions dans l'espace vécu, dans les façons d'habiter (Veschambre 2005).

Aborder ces villes par le biais du patrimoine, dans le contexte néolibéral, suppose deux choses :

-que l'on considère le patrimoine industriel comme partie intégrante des objets patrimonialisés, c'est-à-dire comme appartenant au bien commun,

- et cependant qu'habiter le patrimoine industriel a ceci d'original qu'il s'agit d'un patrimoine récent, lié au travail et aux conflits qui en découlent, lié aussi à la violence contemporaine des suppressions d'emplois massives et des fermetures d'unités de production.

Aussi interroger le patrimoine industriel comme élément de revitalisation ne peut faire l'impasse sur la persistance de ces conflits, mais doit aussi se concevoir comme un moyen de refuser la mort des territoires. En cela, la question de la patrimonialisation, dans ces territoires industriels, perdure comme une problématique politique et sociale forte, modifiant les fonctions urbaines et/ou s'adaptant à leurs modifications.

Notre propos, ici, abordera la spécificité de la patrimonialisation du bâti ou des outils à la fin de l'activité industrielle, puis la fonction sociale de la patrimonialisation parfois bien après la désindustrialisation. Enfin la revitalisation par la mise en tourisme peut être à l'origine d'une nouvelle appropriation du patrimoine.

\section{Passer des ruines, de la friche au patrimoine}

\section{A. Un patrimoine à part}

5 Le patrimoine impose un rapport de proximité. Le patrimoine industriel parce qu'il est encore récent, est sans doute plus que tout autre constitutif de l'identité du territoire, et ceci d'autant plus que le territoire s'est constitué autour de l'activité de production. Il profite de l'élargissement de la notion patrimoniale, à la triple extension typologique, chronologique et géographique des biens patrimoniaux et de la consécration de monuments appartenant à un passé sans cesse plus proche (Choay, 1992). Les éléments $\mathrm{du}$ patrimoine industriel, comme tout autre, sont, par définition constitutifs d'un héritage à transmettre aux prochaines générations, et que l'on tâche de mettre hors du temps. La notion de patrimoine industriel, en France, naît en 1976 dans les textes d'un colloque qui se tient au Creusot, mais ce n'est qu'en 1983 qu'est créée une cellule de patrimoine industriel auprès de l'inventaire des monuments et richesses artistiques de la France. Malgré tout, la création de cette cellule ne garantit pas toujours de bonnes dispositions des pouvoirs publics quand le contexte semble imposer d'autres priorités, notamment économiques.

Selon le premier article de la Charte de Nizhny Tagil pour le patrimoine industriel, publiée en juillet 2003 à l'initiative du TICCIH (The International Committee for the Conservation of the Industrial Heritage), «le patrimoine industriel comprend les vestiges de la culture industrielle qui sont de valeur historique, sociale, architecturale ou scientifique. Ces vestiges englobent : des bâtiments et des machines, des ateliers, des moulins et des usines, des mines et des sites de traitement et de raffinage, des entrepôts et des magasins, des centres de production, de transmission et d'utilisation de l'énergie, des structures et infrastructures de transport aussi bien que des lieux utilisés pour des activités sociales en rapport avec l'industrie 
(habitations, lieux de culture ou d'éducation)». Cela suppose donc que le patrimoine industriel prenne en compte les glissements de modes d'habiter les lieux, les métamorphoses des appropriations des territoires urbains et usiniers, leur fragmentation.

7 Toutes les friches industrielles, comme les a nommées B. Mérenne-Schoumaker (Bruyelle et Mérenne-Schoumaker, 1992), ont en commun d'être la manifestation d'un abandon, d'une perte de la fonction première, le témoignage d'une crise. La friche marque la fin d'une territorialité, d'interrelations entre la ville et l'espace productif, matérialisée par des espaces en marge mais offrant aussi de nouvelles possibilité d'aménagement (Andres et Janin, 2008). Elle est le résultat des renouvellements de formes de production et d'emplois, des principes de localisation, et avec, de la répartition mondiale des activités. Ce patrimoine industriel est donc un patrimoine à part, associé à une vie sociale ouvrière en berne, à un dynamisme économique révolu. Ce bâti est aussi souvent mal accepté, vu comme une dépréciation du paysage, une perte sur les plans esthétiques, économiques, écologiques et n'est pas toujours perçu comme un patrimoine, difficulté d'autant plus grande qu'il s'agit parfois d'objets " gigantesques et encombrants" pour reprendre le titre du document du TICCIH.

La question de la sauvegarde du bâti et de l'outil industriels ne s'est donc posée que très récemment et de façon disséminée. Si aujourd'hui cette démarche, dans la plupart des cas, semble aller de soi, ce n'était pas, loin s'en faut, une évidence aux yeux des aménageurs des villes en pertes d'industrie. En effet, une réaction fréquente s'est traduite par la destruction des usines en friches et la volonté de faire disparaître toute trace de l'économie industrielle de la ville qui venait d'échouer et d'emporter avec elle des centaines d'emplois, selon un principe qui veut que tout ce qui n'est plus utile à la production n'est plus souhaitable parce que nuisible à une image, ou à l'origine de dépenses inutiles. Ainsi, fréquemment le bâti devenu friche est démoli, le terrain nettoyé, aplani pour de nouvelles utilisations. Le paysage est alors considéré comme "assaini». Il existe donc une forme de conflit apparent entre revitalisation économique d'une part et la patrimonialisation d'un passé industriel d'autre part.

\section{B. Un patrimoine objet de conflits}

\section{Refuser la mort et commémorer ou détruire et tourner la page}

9 Sauvegarder l'héritage industriel est une question qui n'est abordée, en France (plus d'une décennie plus tôt à l'étranger, notamment au Royaume-Uni), qu'au début des années 1970. Elle s'illustre notamment par la naissance de l'écomusée du Creusot en 1973. En effet, à la création de l'écomusée, il s'agit d'inventorier le bâti, le matériel et de sauver les bâtiments de production et de logement de la destruction. Or, après la faillite de Creusot-Loire en 1984, l'urgence est à l'absorption du choc et au deuil des emplois et de ce qui a caractérisé la ville. Il n'est alors pas question, pour les pouvoirs publics, d'entendre parler du passé. À ce titre, un conflit est exemplaire: en 1985, la Communauté Urbaine du Creusot-Montceau les Mines achète le terrain de la Plaine des Riaux. S'agissant du site de la première fonderie, lieu de la première coulée, l'écomusée fait une demande de fouilles, mais celle-ci est vécue, interprétée comme un risque de retard sur la reconversion. La priorité économique est invoquée, il ne s'agit pas du tout de parler de l'histoire glorieuse de la ville dans une période de crise violente. Il en sera aussi plus durablement le cas dans le bassin minier du Nord-Pas de Calais avant de 
concevoir la valeur symbolique d'objets paysagers comme les chevalements, ou les terrils. Cette phase de deuil (Grossetti M. et alii, 1998) semble d'autant plus destructrice qu'elle est longue et que l'oubli fait son chemin, déliant l'identité locale à l'industrie.

L'alternative pourrait se jouer entre effacer toute trace industrielle d'une ville à l'image perçue comme trop noire et faire du bâti industriel le support d'un renouvellement économique et culturel B. Lusso l'évoque entre Manchester et la Vallée de l'Emscher (Lusso, 2010). Une étape entre ces deux extrêmes prend corps dans des forme de préservation ponctuelle de type "sauvegarde-alibi ", évoqué par Woronoff, réalisées " pour se donner bonne conscience ou faire une concession à l'esprit du temps » ou par $\mathrm{V}$. Veschambre: «on veut bien conserver des héritages industriels mais en nombre limité, dans des espaces circonscrits, pour que cela ne soit pas trop coûteux et compliqué à gérer » (Veschambre, 2008, p.174).

11 Ce patrimoine est vécu diversement et ceci parce qu'il s'agit avant tout d'un patrimoine construit sur un manque, une ablation d'un élément essentiel de l'habiter précédent. Il pourra être, par la suite, un support de revendication d'une identité et d'une fierté, donnant lieu, par exemple, en juillet 2012 au classement du bassin minier du Nord-Pas de Calais sur la liste du patrimoine mondial évolutif de l'Unesco, au titre de paysage culturel évolutif. Sylvie Daviet évoque cette alternative à propos de La Ciotat et la Seyne-sur-Mer après la fermeture des chantiers navals : « Dans ce long processus, les luttes sociales peuvent donner lieu à des lectures contradictoires, mais traduisent une question de survie: le refus de la mort n'est pas un archaïsme. La mémoire et le patrimoine comptent. La Seyne qui a rasé ses chantiers n'a pas avancé plus vite. L'identité industrielle, comme toutes les identités reste ambiguë, elle peut être repli, fermeture, ou au contraire porteuse de renouveau » (Daviet, 2005, p. 53). La survie du patrimoine industriel repose alors moins sur les systèmes de protection, certes indispensables au départ, que sur la création de ce qu'on pourrait appeler un «lien social» fort entre une population, une société, et les survivants de ce patrimoine.

\section{Mémoire des conflits de classe et leur transmission}

12 Après les années 1970, la conservation du patrimoine industriel, devient, dans certains cas, une préoccupation collective. Lorsque la patrimonialisation est décidée, il reste à savoir ce qui doit être conservé et comment. De cela dépendent aussi l'appropriation du patrimoine dans ses nouveaux usages, et les façons de l'habiter, d'y accéder, d'en faire un lieu de plus ou moins grande quotidienneté. En effet, la question de l'habiter ne se restreint pas à une question de proximité (l'humanité n'a pas d'abord vécu dans le proche pour ensuite s'ouvrir à la mobilité), de petite échelle (les individus organisent leur mode d'habiter à travers une pluralité d'échelles) et de milieu (concept qui manifesterait une connotation biologique, et présenterait donc un risque de déterminisme), elle porte néanmoins la notion d'une mémoire collective. En ce sens le patrimoine est témoignage et transmetteur des conflits de classe inhérents à la production et au travail industriel. Maurice Halbwachs évoque le travail matérialisé comme mémoire commune différente de l'histoire (Halbwachs, 1997). On peut considérer, dans ce contexte, le patrimoine industriel comme une fossilisation du travail. Sa mise en valeur et le choix de l'héritage à transmettre est ensuite fonction de la démarche, selon qu'elle peut être qualifiée de « top down » ou de « bottom up ». 
13

trons le propos par l'exemple du patrimoine industriel minier. Celui-ci est devenu éminemment politique dès le début des fermetures des fosses et jusqu'aux années 1990 les initiatives de conservation sont quasi uniquement patronales autour d'un élément central : la Fosse Delloye à Lewarde, fermée en 1971. Le Centre historique Minier de Lewarde est donc installé par les Houillères du Bassin du Nord-Pas de Calais (HBNPC). Relativement isolé au sud de Douai, cet ensemble n'a ni coron, ni terril, ni industrie annexe, ni chemin de fer. Il présente le travail minier sous un aspect technique, scientifique et pédagogique avec la reconstitution en surface d'un «parcours du mineur » dans une galerie.

Quelques initiatives de mobilisations voient le jour mais ne bénéficiant pas des mêmes financements, leur lisibilité est bien plus restreinte. Il faut attendre les années 1990 pour que les mobilisations de conservation existent à tous niveaux, notamment à celui d'instances publiques et organisations syndicales. C'est le cas d'Oignies où la municipalité a favorisé avec les syndicats la conservation de la fosse 9-9bis où a été découvert le charbon en 1849 et d'où est remontée la dernière berline du bassin le 20 décembre 1990.

Les exemples miniers soulignent le contraste entre l'initiative patronale (top down) dont a hérité politiquement la région et les initiatives locales de générales et d'associations (bottom up) qui ont refusé le monopole de principe envisagé par les HBNPC. Sans grande surprise ce sont les hommes, mineurs, ouvriers, leur savoir-faire mais aussi leur histoire, leurs luttes qui prennent le plus de place dans la valorisation patrimoniale d'origine non entrepreneuriale. C'est notamment le cas du site de la catastrophe de Courrières situé à Méricourt, Billy-Montigny et Sallaumines. Le 10 mars 1906, un coup de grisou suivi d'un coup de poussier dévaste $110 \mathrm{~km}$ de galeries dans les fosses 2 à Billy-Montigny, 3 à Méricourt et 4-11 à Sallaumines et tue 1099 mineurs. La gestion de catastrophe par les autorités minières et locales d'alors, privilégiant, 2 jours seulement après la catastrophe, la remise en état des galeries plutôt la recherche d'éventuels survivants, provoqua une crise sociale et politique de plus deux mois, violemment réprimée. L'inauguration, en 2006, du parcours des 13 rescapés (photo 1), sortis 20 jours après la catastrophe, montre la vivacité de cette mémoire ouvrière dans ces territoires. Ainsi la patrimonialisation fait plus que cristalliser des identifications, des solidarités ou des conflits, elle est transmission mais aussi résistance sociale. C'est ce que Maurice Halbwachs a nommé "mémoire collective ", c'est-à-dire la "faculté collective de se souvenir » entre comme l'un des éléments principaux du processus de résistance sociale à l'œuvre lors des conflits sociaux. Les traces qui demeurent ensuite font partie intégrante de l'identité du patrimoine social urbain. Pourtant, force est de constater que l'époque actuelle, par le biais de la globalisation, tend à nouveau, à favoriser les modes de patrimonialisation «top down ", introduisant alors jusque-là une forme de standardisation dans la valorisation patrimoniale. 


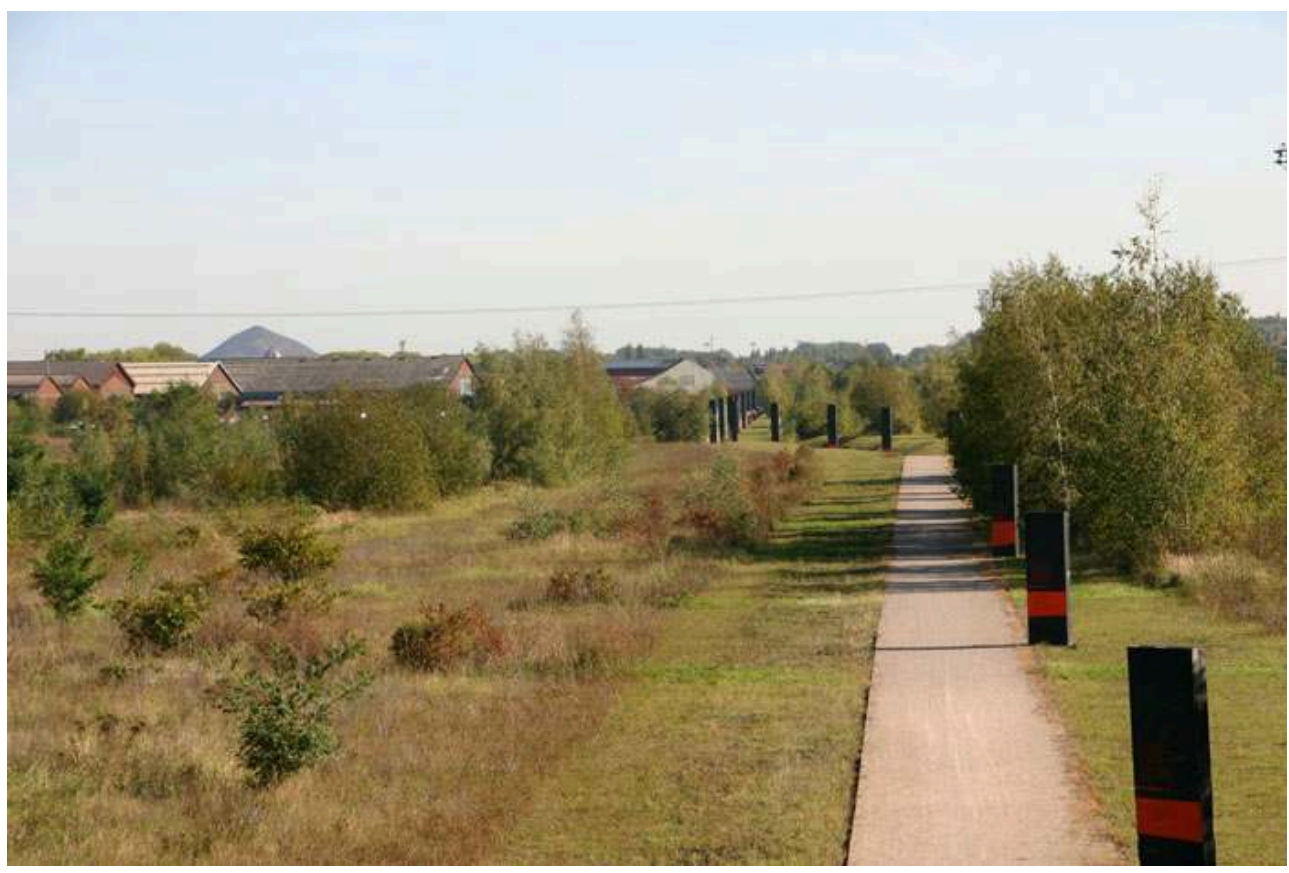

\section{Patrimoine et palimpseste urbains}

Néanmoins même porteur d'une forte identité commune, d'une histoire riche, un lieu n'est pas intrinsèquement patrimonialisable. La patrimonialisation est un processus socio-spatial non exempt de choix idéologiques. Le patrimoine industriel n'existe que s'il en est décidé ainsi, et cette décision politique en fait alors une nouvelle construction sociale avec un biais interprétatif. Un axe de réflexion peut être de traiter ce patrimoine industriel comme n'importe quel autre en le conservant sous forme de ruines. Les Jardins des Terrasses (photo 2) au Creusot en sont un exemple par leur mise en scène urbaine. A la fin de l'activité de Creusot-Loire, les hauts-fourneaux ont été détruits. Ce qui reste de la démolition est laissé à l'état de vestiges sur les terrasses dominant la Plaine des Riaux et la halle aux grues et locomotives, tout comme est conservé le tunnel qui permettait l'arrivée de l'acier pour l'usinage. Le choix de conserver en l'état ces éléments est contemporain de la réflexion architecturale de Banlieues 89 , où le paysage est appréhendé à travers un prisme culturel. Cet aménagement permet de prendre conscience des volumes de ces hauts-fourneaux, mais n'implique aucune disposition particulière pour empêcher le reste du bâti de tomber définitivement en ruines au passage du temps. La conservation de ce site, visible, accessible à tous, est une illustration de ce que le paysage urbain peut rendre et laisser imaginer. Contrairement à d'autres sites le choix qui est fait au Creusot n'est pas le résultat, par défaut, d'un manque de moyens mais bien d'un parti pris. Notons toutefois qu'une telle démarche reste, en France, rare concernant le patrimoine industriel, elle est davantage utilisée pour la mise en valeur de vestiges d'époques plus anciennes. 
Photo 2 : Ancien haut fourneau. Jardins des Terrasses au Creusot. 2006

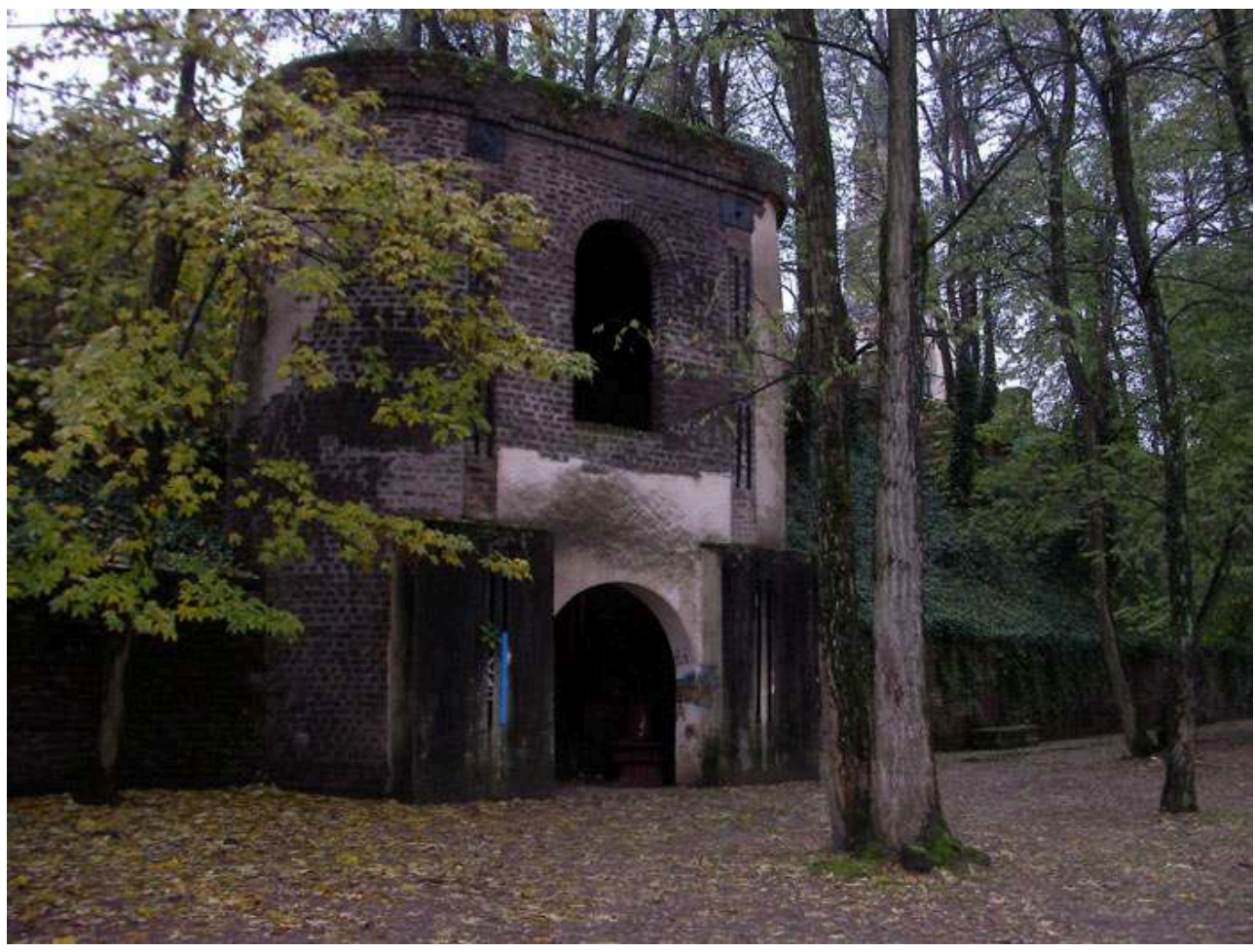

Plus classiquement il s'agira de muséification comme le centre de Lewarde déjà évoqué, comme le carreau de la mine de Wallers-Arenberg, résultat, lui, de la détermination des élus locaux des communes de la Porte du Hainaut, ou encore comme l'Académie Bourdon au Creusot valorisant les savoir-faire techniques. Le cas des mines-images est intéressant sur ce point. Du temps du fonctionnement des mines, les mines-images sont des centres de formation pour les mineurs de fond, reproduisant en béton des galeries de mines, avec des salles de cours et des salles de mécanique. Ce sont aujourd'hui ces lieux qui sont le plus facilement convertis en musée comme celui de la Mine-Image à La Motte-d'Aveillans, site d'extraction d'anthracite des mines de La Mure, au sud de Grenoble. L'exploitation de ce site a débuté de façon artisanale au XIVe siècle, avant d'être industrialisée au XIXe siècle. L'exploitation minière sur le site mottois prend fin en 1956, puis les mines sont fermées en 1997 après deux siècles d'extraction, mais le projet de musée est porté dès 1987 avec l'association Sauvegarde regroupant d'anciens mineurs. Il regroupe deux sites : celui de la Mine-Image à La Motte-d'Aveillans et le Musée Matheysin à La Mure.

Une autre démarche encore est celle d'inscrire la mémoire dans la reconstruction de la ville sur la ville. C'est le cas par exemple de l'ancien site des usines ETG-Chausson à Gennevilliers. L'activité industrielle s'y est arrêtée en fin d'année 2007, le site a ensuite été rasé puis dépollué. Le projet d'aménagement comprend un écoquartier, fonctionnellement mixte, mais les acteurs publics et politiques locaux (municipalité, anciens salariés, société d'histoire de Gennevilliers) ont souhaité y insérer une trace du contexte urbain préexistant à travers l'installation de «boites reliques » contenant des objets de l'usine, pour marquer, dans le sol de ce nouveau morceau de ville, l'ancien périmètre de l'usine. Il ne s'agit pas ici d'une forme de "patrimoine-alibi ", évoqué plus haut, cherchant le moindre coût ou la bonne conscience, mais d'une démarche de reconstruction de la ville sur elle-même sans rien oublier du passé industriel qui a pu 
être à l'origine de son développement. La patrimonialisation ainsi envisagée refuse de freiner l'évolution et de figer le paysage urbain.

Chacun de ces exemples donnent de manière différente des orientations de patrimonialisation, de valorisation, tout en permettant, favorisant l'appropriation du patrimoine. La reconversion des friches s'inscrit dans le renouvellement de la ville en préservant une part plus ou moins importante de la couche industrielle du palimpseste.

\section{Une question sociale : habiter le patrimoine industriel}

\section{A. Un espace vécu en transition}

20 Le patrimoine industriel est une question sociale par la décision qui est prise de le conserver ou non, il est aussi une conception sociale car il n'existe que dans des appropriations et des configurations sociales et historiques particulières. Ainsi, la conservation du patrimoine industriel ne remplit sa fonction qu'en étant force d'évocation collective dans la ville, pour l'ensemble de la population, c'est-à-dire seulement si ce patrimoine participe de la transmission de l'identité commune. Cela passe bien sûr par le paysage qui peut être considéré selon l'expression de Guy Di Méo comme « une fenêtre ouverte sur le territoire »; mais aussi par la perception que l'on en a et par les façons d'habiter l'espace et de porter cette identité.

21 Le paysage de la ville-usine ou de la ville minière est ordonné selon un urbanisme fonctionnaliste. Cette fonctionnalisation industrielle de la ville, par le processus de désindustrialisation actuel, conduit à la remise en cause de son urbanité, c'est-à-dire à la perte de tous les avantages liés à la fonction de centralité, à celle d'espace public, à ce qui fait la ville. Il n'en est pas de même pour les villes préindustrielles pour lesquelles la variété de fonctions préserve le caractère urbain. La mémoire de l'ensemble de la population des villes industrielles permet donc de parler d'une identité qui se territorialise sur l'espace public. Le passage de ce qui est de l'ordre de l'espace vécu comme lieu de travail, au «lieu de mémoire» (Nora, 1997) collectif, est lui aussi une marque de la désindustrialisation et contribue à faire de ces villes une interface temporelle mais aussi identitaire.

Le paysage industriel est donc partie intégrante de l'identité urbaine, elle-même reposant sur l'identité des groupes sociaux qui la composent. Le vécu industriel de la ville, passé ou présent, passe par cette mise en commun de l'expérience à l'intérieur de l'usine et à l'extérieur, par chacun des éléments qui aura rythmé la vie autour de l'espace usinier (Di Méo, 2007). Ainsi, peu à peu, la ville industrielle devient une ville de traces, qu'il subsiste longtemps des slogans, comme à Gennevilliers, sur l'un des murs de l'enceinte de l'usine ETG Chausson, ou des fresques revendicatrices comme celles du Creusot (photo 3). 
Photo 3 : Extrait de l'une des fresques du Creusot peinte après le démantèlement de Creusot-Loire au milieu des années 1980. 2006

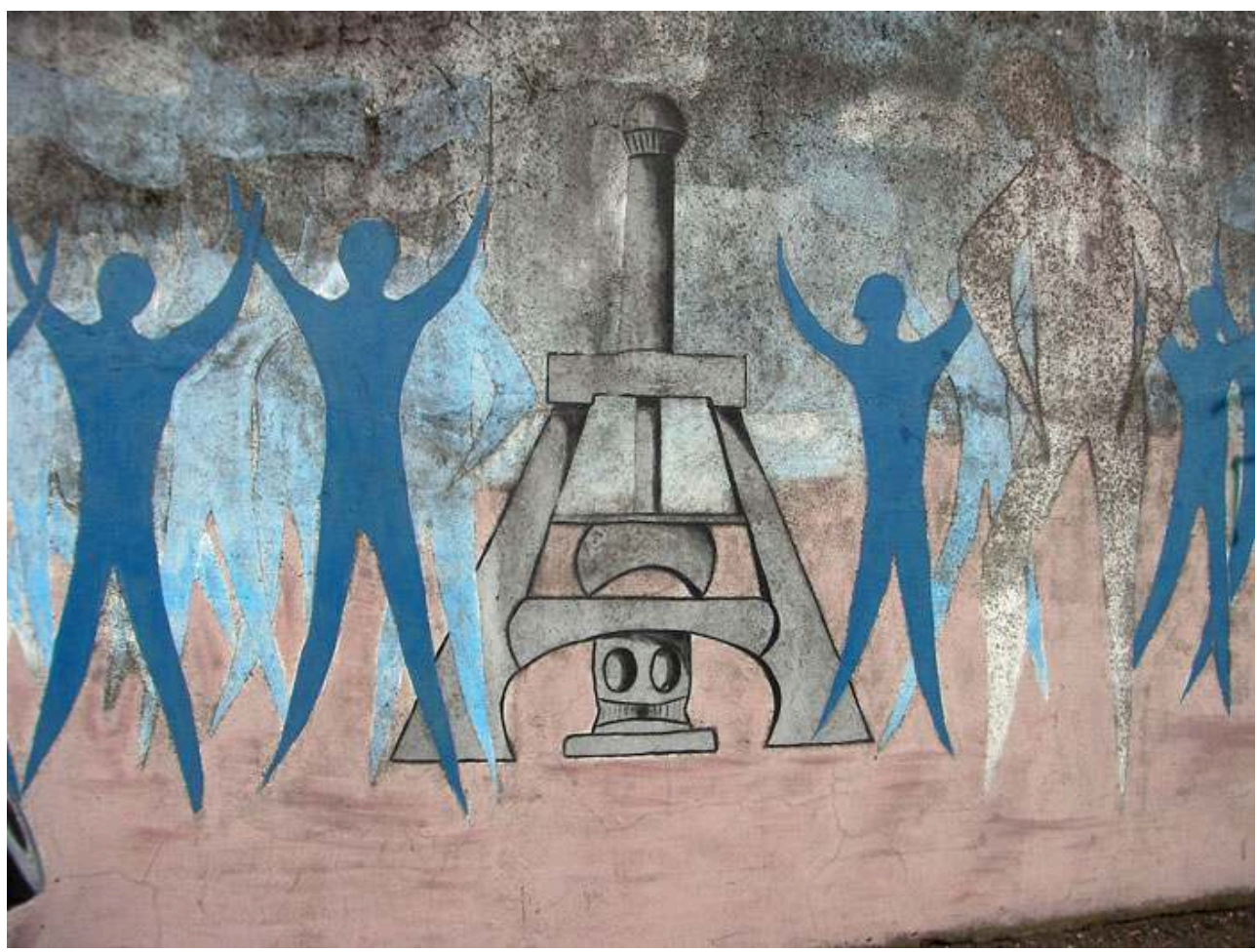

L'espace est chargé de valeurs sociales, de symboles, d'imaginaires. En cela, il est le produit d'une société, de ses représentations spatiales, de ses sentiments d'appartenance. Il est à la fois réceptacle d'identité et le lien entre cette identité et la société, au sens où il est un espace vécu, défini comme l'ensemble des lieux fréquentés par une personne ou par un groupe ajouté aux interactions sociales qui le sous-tendent et aux valeurs psychologiques qui s'attachent aux lieux et qui unissent les hommes à ceux-ci et par les liens matériels.

Si l'on considère que la conservation du patrimoine industriel participe de l'identité d'une ville, alors elle est partie prenante de son aménagement, de son environnement et de la définition de nouvelles fonctions urbaines. La structuration des paysages industriels fait ainsi partie du patrimoine, en effet, les friches, selon le traitement dont elles font l'objet peuvent être considérées comme des éléments potentiellement constitutifs d'un nouveau patrimoine et par conséquent d'un nouvel environnement. C'est l'un des enjeux qui peuvent exister autour des possibilités de faire cohabiter patrimoine industriel et nouvelles formes d'aménagement, de préserver un lien historique et social dans la ville à partir des différentes occupations précédentes de l'espace et enfin de recoudre les éléments du tissu urbain.

\section{B. Habiter la ville industrielle : particularité du logement}

Nous avons déjà noté le peu d'attention porté traditionnellement au patrimoine d'origine industrielle, malgré la force des imaginaires et des identités qu'il soutient. Si certains lieux emblématiques de production ont été conservés et réaffectés à d'autres usages culturels pour l'essentiel, fort peu de logements construits par les industriels font l'objet de mesures de protections ou de conservations, au vu de la considérable 
production urbaine d'essence hygiéniste et paternaliste de la fin du XIXe siècle et au début du XXe siècle. A travers l'invention du quartier ouvrier, la moralisation de la vie familiale par le logement social rejoint la normalisation du travail que constitue la condition salariale, en même temps qu'il joue un rôle non négligeable de pacification sociale (Topalov, 2002) et il est révélateur que les bâtiments conservés - avant même les usines et les cités - sont les bâtiments dits de "subsistance ", c'est-à-dire ceux construits pour la vie quotidienne des ouvriers: écoles, crèches, foyers, hôpitaux, stades, etc.. Sur ce point, Vincent Veschambre rappelle, qu'appliqués aux espaces industriels, les choix de conserver ou d'oublier des lieux dédiés à la production ou à la reproduction de la force de travail ne sont pas neutres. Vouloir conserver au titre du patrimoine industriel les cités ouvrières, productions reconnues comme paternalistes et dont les murs peuvent être la représentation d'un espace patronal avant d'être celle d'une sociabilité ouvrière, n'est pas forcément antinomique si l'on considère que " l'usage prescrit par les formes construites n'engendre pas forcément les conduites attendues ", autrement dit que ces cités sont aussi ce que leurs habitants en ont fait et en font en se les appropriant (Söderstrom, 1997, p. 178).

Photo 4 : Cité-jardin à Stains. 2012

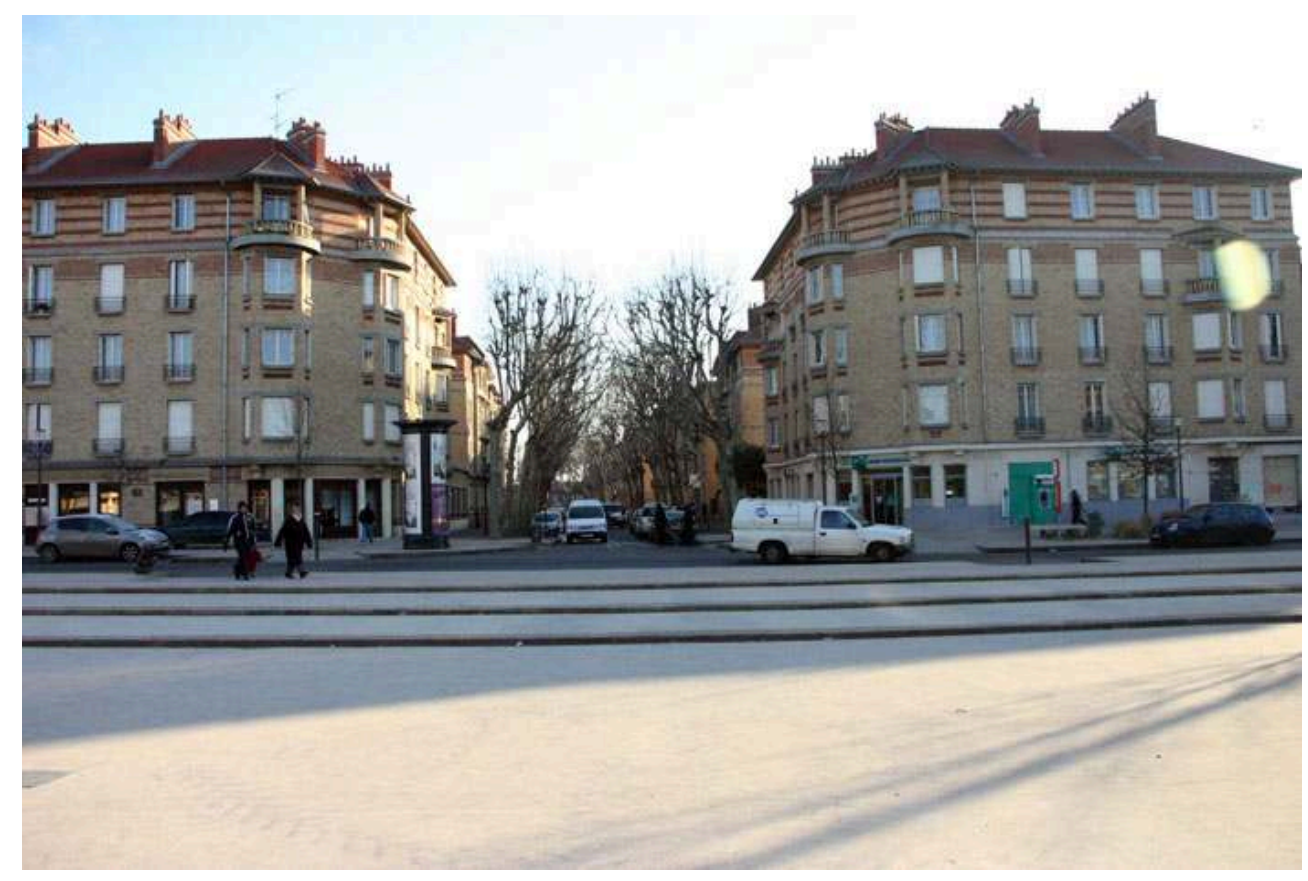

Considérant l'empreinte paysagère, elle varie selon les constructions et leur contexte. Le Creusot fournit un exemple de cette structuration et identification par la forme de l'habitat. Ainsi les différenciations sociales sont visibles et inscrites dans le paysage urbain, notamment par la couleur des toitures des maisons, en ardoise pour les maisons d'ingénieur, ou les bâtiments relevant de la hiérarchie d'encadrement ou patronale, en tuile rouge pour les maisons d'ouvriers. Les fonctions de logement, de production, d'apprentissage par exemple sont aussi largement perceptibles dans l'organisation de la ville. L'évolution du profil de la ville n'a pas remis en cause cette disposition qui alors persiste à imprimer le territoire. La patrimonialisation des cités ouvrières, qu'elles soient sous la forme de cités-jardins (photo 4) principalement en région parisienne ou à Bourges, à Reims ou sous forme d'ensemble comme la Cité de la Combe des Mineurs au 
Creusot ou les cités du bassin minier du Nord-Pas de Calais renvoient à la nécessité pour un groupe social d'être reconnu. François Duchêne l'explique ainsi : «En d'autres temps, le recours à la patrimonialisation par le classement de nombreuses propriétés et châteaux en monuments historiques, a permis à un groupe social se sentant tout aussi menacé dans son statut et dans ses bien d'être "restauré dans un équivalent symbolique de son statut d'origine ". A cette différence près que la noblesse du XIXe siècle pouvait encore faire alliance avec les groupes dominants de son époque, contrairement aux ouvriers d'usine du XXIe siècle». (Duchêne, 2005, p. 617-526). Ainsi, de la même façon qu'à travers le classement des quartiers ouvriers, des outils, des machines, sont mis en scène afin d'inscrire le rappel de cette mémoire commune dans l'espace, le paysage.

\section{Revitalisation, amputation, confiscation, réappropriation}

\section{A. Revitalisation}

La patrimonialisation n'est pas uniquement conservatrice, voire dans certains de ses aspects, pétrifiante. Les grandes installations en friches peuvent accueillir de nouvelles activités industrielles ou bien être réutilisées par le biais de solutions non industrielles. En dehors des solutions anecdotiques de transformations en loft, la revitalisation de certains bâtiments aux caractéristiques architecturales remarquables peut en faire des lieux d'usage public. Citons pour exemple, la reconversion de la halle aux grues et aux locomotives du Creusot en bibliothèque universitaire. Son réaménagement est réalisé dans un principe de réversibilité. Le bâtiment ainsi réinvesti possède une double évocation dans le paysage urbain : celle du patrimoine industriel, et celle de l'actualité estudiantine. Le principe de revitalisation de dents creuses n'est pas neuf. Il opère souvent pour la reconversion en lieux culturels, comme pour la Manufacture des CEillets à Ivry-sur-Seine, la très récente ouverture du Métaphone à Oignies sur le site de la fosse 9-9bis ou la Friche de la Belle de Mai à Marseille. Cette reconversion ne rencontre, pour l'heure, pas de polémique équivalente, à ce que l'on trouve au Royaume-Uni où il est souvent reproché d'utiliser la reconversion culturelle de façon trop systématique (Bailoni, 2008).

Le cas de cette friche est particulier. Ancien site de la Manufacture des tabacs de la Seita, son investissement par un projet artistique a permis une revalorisation du site, en termes d'image mais aussi foncière et urbaine, incluant aujourd'hui, outre le complexe artistique, des équipements publics et des logements sociaux afin d'amorcer une couture urbaine entre le site de la Friche de la Belle de Mai et le quartiers des anciens salariés de la Seita. La Friche est devenue l'un des projets clefs du vaste projet de régénération Euroméditerranée tout en maintenant une volonté de rejet des dynamiques de gentrification à l'œuvre dans les autres quartiers concernés par l'Opération d'Intérêt National. C'est aussi un lieu porteur de Marseille-Provence 2013 capitale européenne de la culture. Sur ce site, les temporalités de la patrimonialisation sont différentes, notamment parce que l'acceptation de départ est que les usages, notamment culturels, qui sont faits de ce site sont temporaires (Andres, Grésillon, 2011). Bien entendu nous ne sommes plus là dans le cas d'une ville spécifiquement développée ou formée autour de l'industrie et les moyens, les objectifs, le contexte sont ceux d'une ville de près d'un million d'habitants, néanmoins cet exemple permet 
d'envisager d'autres dynamiques que la seule et très (trop ?) fréquente reconversion en lieu culturel.

\section{B. Dépossession, pétrification et conflit mémoriel}

29 La patrimonialisation dans sa forme de muséification, qu'elle obéisse à une volonté militante locale ou à une démonstration de savoir-faire et de savoirs techniques, porte en elle une charge affective, une lecture du passé. La réinterprétation du bâti sous d'autres formes, répondant aux injonctions d'un présent plus tourné vers le tertiaire tend à commuer le passé en objet de consommation, de spectacle. Ce faisant la réaffectation du bâti industriel implique une pacification de la mémoire, rendue propre à consommer, ou bien parce que les nouvelles activités n'auront plus rien à voir avec les activités précédentes ou bien parce que la muséification aura pétrifié le témoignage de l'héritage industriel.

Le changement de fonction de l'édifice entraîne la modification du regard et de sa perception par la population. Sa présence dans l'espace public n'a plus le même caractère. Ce sentiment est amplifié lorsque le bâtiment industriel, comme parfois les quartiers ouvriers, sont gentrifiés au point de n'avoir plus que très peu de rapports avec son identité première. En effet, la visibilité de l'objet industriel mis en scène de cette sorte est dépendante de la démarche que fera l'usager, qu'il s'agisse d'un musée, comme celui de Lewarde, ou des usines Menier de Noisiel, converties en siège social de l'entreprise Nestlé, mais dont l'image est utilisée comme identité et symbole du savoirfaire. On peut alors parler de confiscation du patrimoine ou autrement d'une forme d'amputation de l'identité du territoire. C'est un rapport de différences sociologiques, de sentiment d'appartenance à un groupe plutôt qu'à un autre qui s'immisce ainsi dans le rapport au lieu, dans la façon de l'habiter. En ce sens, l'amputation de l'identité, de l'imaginaire et de la territorialité de l'industrie transcende les classes sociales. Il y a, certes, l'emploi perdu, la difficulté d'en retrouver un, mais aussi la confiscation de l'habiter, de l'espace vécu et avec la part de fierté commune à toute une population.

31 La décision des acteurs publics est là aussi lourde de sens politique. Un espace fortement investi pour son potentiel symbolique véhicule de multiples représentations collectives. L'action publique peut alors ou bien s'inscrire dans le lien, se prononcer sur l'héritage transmis en une action patrimoniale ou bien le nier et assumer son délitement. C'est cette option qui a été retenue lors de l'implantation de la Cité du Design à Saint-Etienne à la place des maisons de direction et des bâtiments administratifs de la Manufacture d'Armes, déclenchant alors un conflit mémoriel et identitaire fort (Zanetti, 2010). S'inspirant nettement, dans la nouvelle fonction, de la Designstadt Zollverein installée sur l'ancien complexe minier d'Essen, la Cité du Design stéphanoise se présente comme son négatif, surimposée au site originel et déliée de son territoire d'accueil.

Enfin dans ce cadre de revitalisation, de réaffectation de fonctions, il est intéressant d'observer comment la tertiarisation de l'époque de l'après-industrie peut favoriser la conservation d'un type de patrimoine. C'est le cas de la friche laissée par la fonderie Vallourec à la limite d'Anzin et de Valenciennes. L'ensemble industriel à fonction productive a été démoli pour permettre l'aménagement d'un nouveau quartier sur les 25 hectares ainsi libérés. Ce nouveau quartier comprend des espaces d'habitations, des activités économiques et des espaces dédiés aux loisirs. L'un des principes retenus pour 
cet aménagement prévoit la conservation de certains éléments paysagers tels que l'immeuble de bureaux de l'ancien siège de l'entreprise métallurgique, c'est-à-dire l'ensemble industriel à fonction décisionnelle.

\section{De nouveaux hauts-lieux pour commémorer}

33 L'alternative possible se joue entre non-lieux et hauts-lieux. Le choix de la mise en scène de nouveaux hauts-lieux définis comme des «lieux qui expriment symboliquement au travers de leurs représentations et de leurs usages, un système de valeurs collectives ou une idéologie » (Debarbieux, 2003), participe donc de la fabrication d'une résonnance identitaire. Il est alors indubitable que des éléments comme les chevalements de mines, les outils, marteau-pilon ou presse à emboutir peuvent constituer des hauts-lieux. Ils ne le peuvent que si le patrimoine est conçu comme pouvant synthétiser cette mémoire commune. A quarante ans d'intervalle, deux communes, Le Creusot en 1969 et Gennevilliers en 2008 ont fait le choix de placer dans l'espace public un outil monumental, qui, lors de son fonctionnement, n'était pas visible. Pour chacune des villes l'outil choisi est porteur de son histoire industrielle et de son imaginaire : pour la première, le marteau-pilon à vapeur rappelle qu'il fut le premier outil à vapeur, pour la seconde, la presse à emboutir électrique Toledo-Bliss des usines ETG-Chausson (photo 5) porte la mémoire de l'un des premiers berceaux de l'industrie automobile française. Tous deux sont des outils imposants - 21 mètres de haut et 100 tonnes pour le marteaupilon, 7 mètres de haut et 155 tonnes pour la presse Toledo-Bliss - et témoignent de la place prise par l'industrie dans la mémoire collective de chacune des villes. La place qui leur est réservée dans l'espace public est similaire, l'un en entrée de ville, l'autre marquant le nouveau centre-ville.

Photo 5 : Inauguration de la Presse Toledo-Bliss à Gennevilliers, 2011

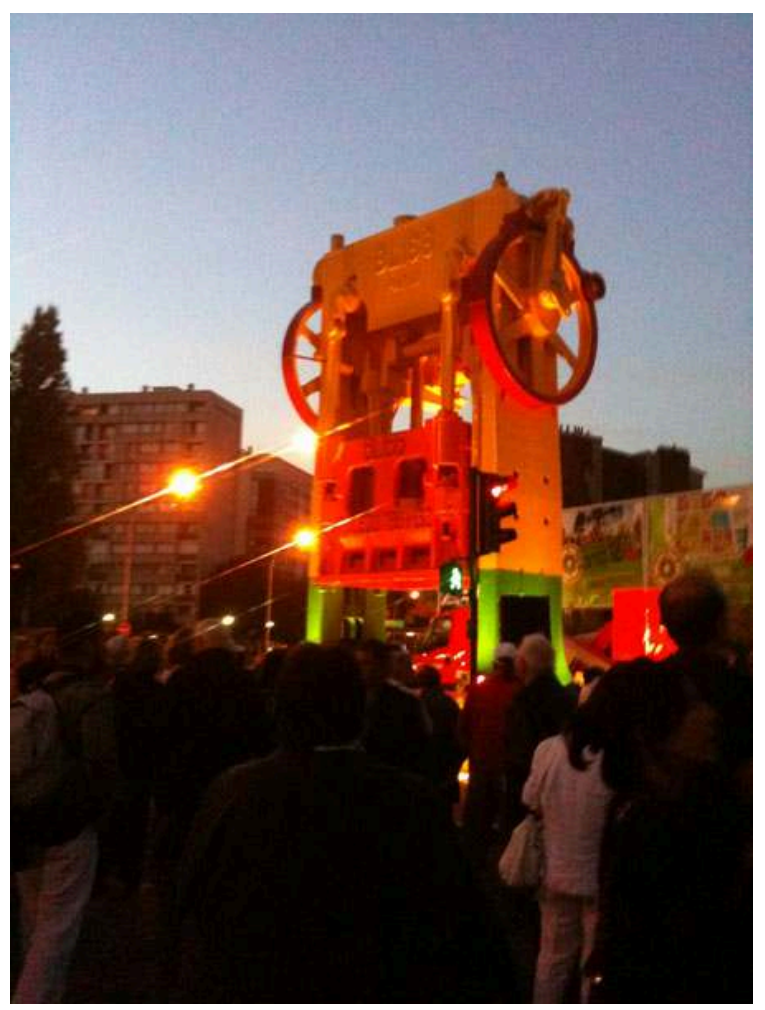

Revue Géographique de l'Est, vol. 53 / 3-4 | 2013 

est marqueur social et révélateur politique de l'attention prêtée à la mémoire collective. Ainsi la dernière vague de patrimonialisation du bâti minier, mettant en scène trois sites, tout juste fermés, la fosse 9-9bis d'Oignies, la fosse de WallersArenberg et la fosse 11-19 de Loos-en-Gohelle répond à un besoin de justifier l'excellence de l'activité, comme celle des travailleurs, mais aussi à la nécessité de gérer le passé et d'assurer un développement durable à un territoire jusque-là synonyme de développement non durable. Il s'agit de dépasser alors les seules "opérations de narrativité du passé» (Barbe, 2003, p. 6), le processus de cristallisation auxquels est soumis tout objet de patrimoine.

À cela, nous pouvons ajouter des œuvres artistiques ajoutées après la désindustrialisation, comme une sorte de monument commémoratif. En région parisienne, la commune d'Ivry-sur-Seine en offre deux exemples, s'appuyant sur les spécificités industrielles de la ville. L'oblique haute de Jean Clareboudt (photo 6), installé en 1991, réalisé en métal rouge-minium, est un rappel à l'importance de la présence de la métallurgie dans cette ville de l'est parisien.

Photo 6 : Oblique haute de Jean Clareboudt à Ivry-sur-Seine, 2008

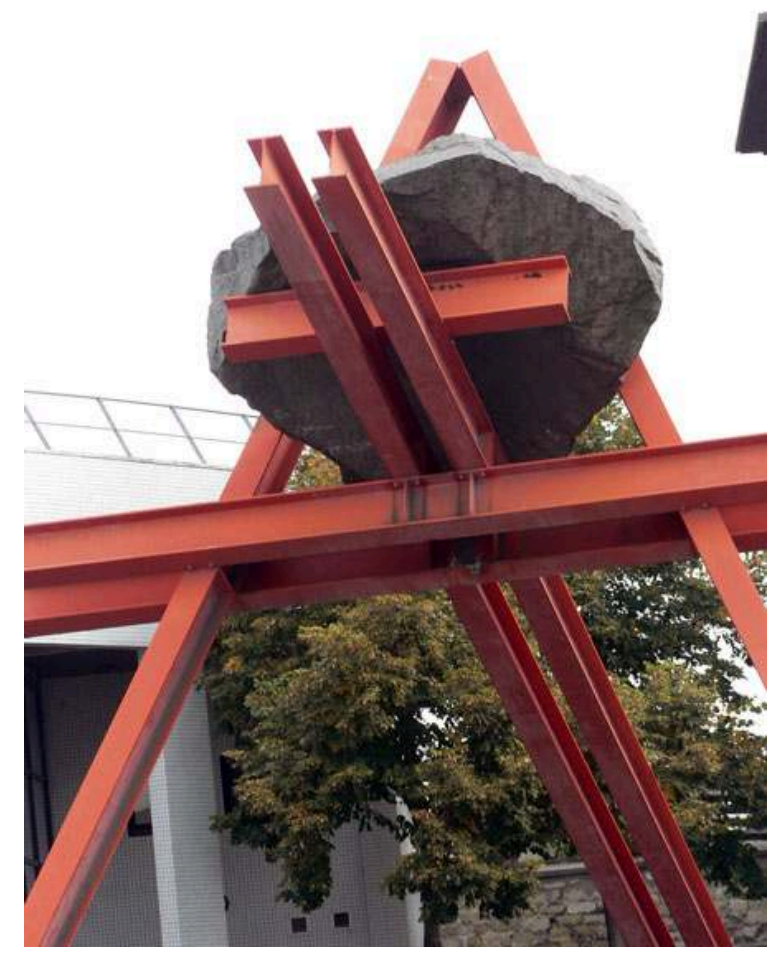

Le Nocturlabe (photo 7), réalisé par Claude Viseux, érigé en 1987 à une entrée de ville d'Ivry-sur-Seine, fait plus qu'œuvre de commémoration de l'activité. En effet, l'implantation du Nocturlabe a été décidée à l'époque de la lutte ouvrière contre la fermeture de l'usine de roulement à billes SKF. L'œuvre utilise la forme des objets produits et la proximité de l'ancien moulin comme en liaison de deux périodes de l'histoire de la ville. 


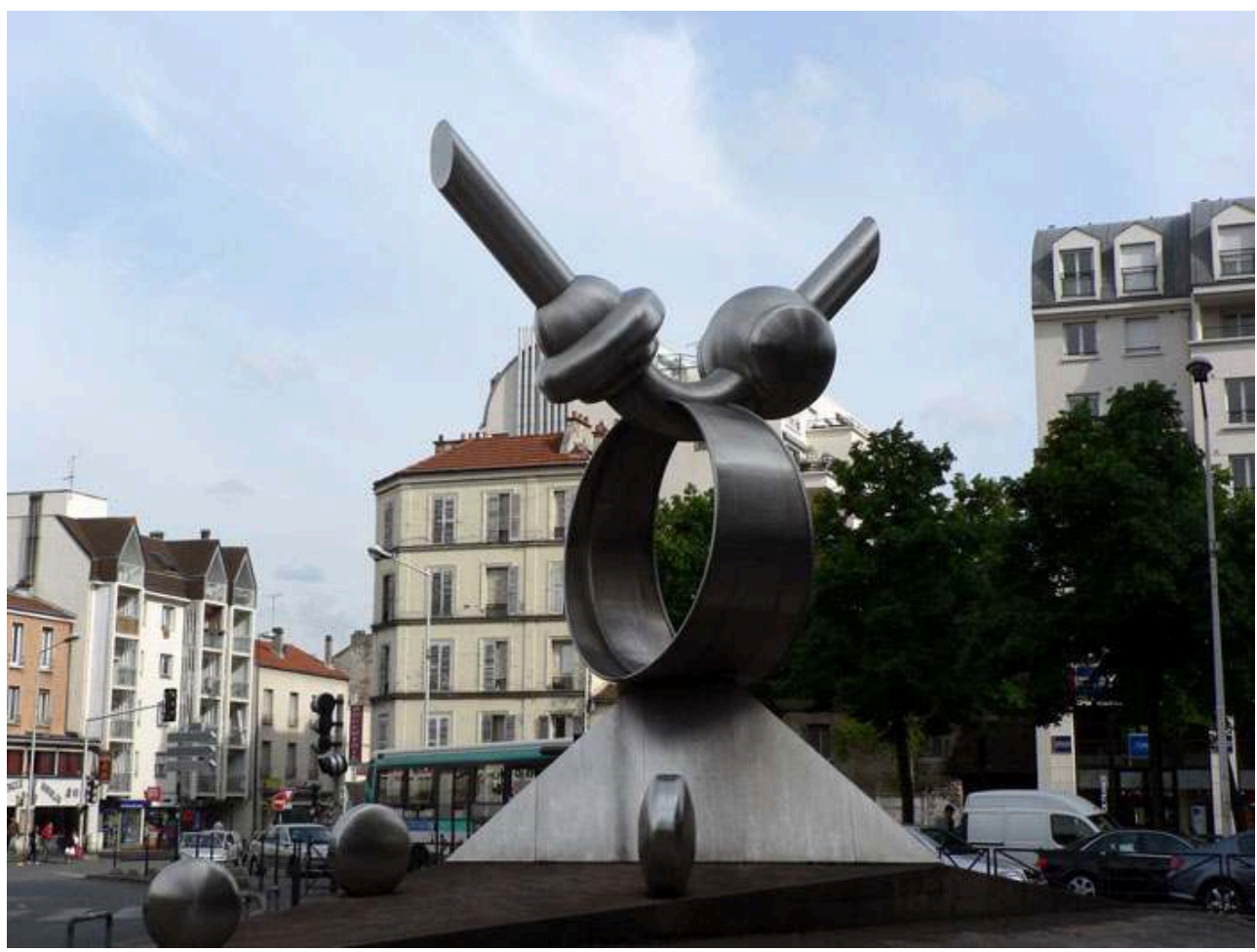

\section{Conclusion}

Pour l'ensemble de ces territoires industriels, l'image du travail est encore fortement ancrée. Il s'agit d'un difficile équilibre politique et social à trouver pour des territoires qui sont loin d'être des territoires morts. Les fragmentations sociales qu'ont connues ces villes ont provoqué réactions, conflits et résistances qui perdurent bien après, conservant aussi une fragmentation territoriale. La mise en valeur de ce patrimoine industriel accompagne alors un refus de mourir, une sorte d'ultime attache à la territorialité avant, peut-être, de symboliser une géographie du manque, de l'ablation.

Sans doute, enfin, que ce patrimoine récent et nombreux ne subsistera pas dans sa totalité, sans doute, ne participera-t-il pas dans son entièreté à une conversion dans l'industrie culturelle, sous forme d'image de marque par exemple, achevant alors les processus de confiscation et de déterritorialisation des identités passées et ouvrant, après l'oubli, sur d'autres pratiques, d'autres mémoires régionales, reconstruites, pacifiées, voire folklorisées pour répondre à une sorte de standardisation touristique et mémorielle. Alors les fonctions urbaines des villes industrielles auront tout à fait muté. 


\section{BIBLIOGRAPHY}

Andres L., Janin C., 2008, « Les friches : espaces en marge ou marges de manœuvre pour l'aménagement des territoires », Annales de géographie, vol. 5, n663, p. 62-81.

Andres L., Grésillon B., 2011, « Les figures de la friche dans les villes culturelles et créatives », L'espace géographique, 1/t40, p. 15-30.

Bailoni M., 2008, «Quelle place pour le patrimoine dans le renouveau d'une région postindustrielle ? Le cas du Nord-Est anglais », Revue Géographique de l'Est, 1-2/2008, URL : http:// rge.revues.org/682

Barbe N., 2003, « Actions patrimoniales », Les Nouvelles de l'archéologie, n93, p. 5-7.

Choay F., 1992, L'Allégorie du patrimoine, Paris, Seuil, coll. La Couleur des idées, 271 p.

Daviet S., 2005, Industrie, culture, territoire, Paris, L'Harmattan, 210 p.

Debarbieux B., 2003, « Haut lieu », in Lévy J., Lussault M., Dictionnaire de la géographie et de l'espace des sociétés, Paris, Ed. Belin, p. 448-449

Di Méo G., 2007, « Identités et territoires : des rapports accentués en milieu urbain ? » Métropoles, (en ligne), $\mathrm{n}^{\circ} 1$, mis en ligne le 15 mai 2007, consulté le 29 mai 2007, URL : http:// www.metropoles.revues.org/document80.htm.

Duchêne F., 2005, Les anciennes cités ouvrières, entre patrimonialisation et normalisation, in Gravari-Barbas M. (dir.), Habiter le patrimoine. Enjeux, approches, vécu, Rennes, Presses Universitaires de Rennes, p. 517-526.

Fol S., Cunningham-Sabot E., 2010, « “Déclin urbain” et Shrinking Cities : une évaluation critique des approches de la décroissance urbaine ", Annales de géographie, n674, Paris, Armand Colin, p. $359-383$

Grossetti M, et alii, 1998, La construction des politiques locales. Reconversions industrielles et systèmes locaux d'action publique, Paris, L'Harmattan, $224 \mathrm{p}$.

Halbwachs M., 1997, La mémoire collective, édition critique établie par G. Namer, Paris, Albin Michel, $295 \mathrm{p}$.

Lusso B., 2010, «Culture et régénération urbaine : les exemples du Grand Manchester et de la vallée de l'Emscher ", Métropoles, (en ligne), n8, mis en ligne le 30 novembre 2010, consulté le 17 décembre 2010, URL : http://metropoles.revues.org/4357.

Söderstromo. (dir.), 1997, L'industriel, l'architecte et le phalanstère. Invention et usages de la cité d'entreprise d'Ugine,L'Harmattan, Paris, $191 \mathrm{p}$.

$\mathrm{TICCIH}, 2000$, Les « dinosaures » du patrimoine industriel. Le gigantesque et l'encombrant sont-ils réutilisables?, TICCIH, document numérique, DVD, ADEC.

Topalov C. (dir.), 2002, Les divisions de la ville, Paris, Ed. de la Maison des Sciences de l'Homme Unesco, $469 \mathrm{p}$.

Veschambre V., 2008, Traces et mémoires urbaines : enjeux sociaux de la patrimonialisation et de la démolition, Presses Universitaires de Rennes, $318 \mathrm{p}$. 
Veschambre V. (2005), Habiter un lieu de production et de travail ; introduction. In : GravariBarbas M. (dir.), Habiter le patrimoine. Enjeux, approches, vécu, Rennes, Presses Universitaires de Rennes, p. 511-516.

Woronoff D., 2007, Quel bilan pour une nouvelle discipline ?, Historiens et Géographes, $\mathrm{n}^{\circ} 398$, p. 132-136.

Zanetti T., 2010, « La Manufacture d'Armes de Saint-Etienne : un conflit mémoriel », Norois (en ligne) vol. 4, n²17, mis en ligne le 31 décembre 2010, consulté le 15 mai 2011, URL : http:// norois.revues.org/index3457.html

\section{ABSTRACTS}

The industrial heritage means that political and social issues are structuring strongly the transformations of urban functions in the industrial cities. Questioning its preservation, the changes of the allocation of the buildings, is therefore beyond the only aesthetic consideration that one could govern the choice to conserve other older buildings. Because of its very recent history, the industrial heritage can't ignore collective identity and memory, and moreover the social meanings of their interpretation. Changes in ways of living and choices of revitalization are then the result of this new role of industrial heritage.

Le patrimoine industriel porte en lui des enjeux politiques et sociaux structurant fortement les mutations des fonctions urbaines des villes industrielles. Le questionnement de sa conservation, des changements d'affectation des divers bâtiments s'inscrit donc au-delà d'une seule considération esthétique qui a pu gouverner le choix de patrimonialisation d'autres bâtis plus anciens. Par son histoire très récente, le patrimoine industriel ne peut pas faire abstraction de l'identité et de la mémoire collective, non plus que des connotations sociales de son interprétation et des conflits de classe qu'elles prolongent. Les modifications des modes d'habiter, les choix de revitalisation en sont alors le résultat.

\section{INDEX}

Mots-clés: ablation, habiter, mémoire collective, patrimoine industriel

Keywords: ablation, collective memory, industrial heritage, live

\section{AUTHOR}

\section{CORINNE LUXEMBOURG}

Maître de conférences en géographie à l'Université d'Artois, membre du laboratoire Discontinuités (EA2468) 